validity (i.e. degrees of success in measurement), as shown by significant relationships between PDQ scores and other variables (Morley \& Snaith, 1989).

(a) PDQ scores have been shown to have predictive validity; for example, in relation to symptoms and signs of depressive disorders (Pfohl et al, 1987).

(b) A degree of construct validity has been demonstrated by factor analysis of PDQ scores in relation to the three clusters of PDs described in DSM-III-R (Dowson \& Berrios, 1991).

(c) PDQ scores have shown concurrent validity in several studies by demonstrating differences between various clinically defined groups; for example, in relation to subgroups of patients with a history of anorexia nervosa (Dowson, 1992).

However, there can be poor agreement between PD diagnosis by questionnaire and structured interview; Hunt \& Andrews (1992) concluded "the PDQ-R may well be tapping some dimension of personality, but it is inappropriate for the assessment of specific personality diagnoses ...". There are certainly discrepancies between PDQ PD diagnoses and clinical concepts of the thresholds for the use of PD diagnoses, as PDQ PDs can be found in 'normal' samples and several studies in patient populations have shown that the PDQ-R has lower thresholds for diagnosis than interview data. But these findings do not necessarily invalidate questionnaire data; cut-off points for PD diagnoses can be modified (although any cut-off point is arbitrary and loses information), while structured interviews are also subject to methodological problems. Perhaps PD is often underdiagnosed by interview methods (i.e. maladaptive patterns of behaviour are missed), particularly in relation to those criteria which do not depend on readily-identified behaviour.

Dr Coid questions the value of an informant's questionnaire when the patient is not compliant. However, the generally poor correlation between patients' and informants' total scores, or between scores for individual PDs, can disguise satisfactory agreement for certain individual PD criteria. But I accept Dr Coid's point that there is no short cut in obtaining a detailed PD assessment in the individual patient and I did not claim that a self-report instrument can achieve this. However, I believe that this format has a research role in groups of patients and, in some respects, such as examining relationships between subtle emotional and attitudinal states, self-report questionnaires may be at least as good as other methods. But, for the individual patient, PDQ self-report data can identify areas for further exploration in the clinical interview, including any obvious discrepancy between the patient's and informant's scores.
The self-report format requires further development (perhaps with scaled ratings for selected items), but has a track record as a research instrument and can complement information from interview assessments. The Screening Test for Co-morbid Personality Disorders (STCPD) also encourages us to look for a range of psychopathology from more than one PD category, which appears to accord with clinical reality in most personality-disordered patients.
Dowson, J. H. (1992) Associations between self-induced vomiting and personality disorder in patients with a history of anorexia nervosa. Acta Psychiatrica Scandinavica (in press).
- \& BerRIOS, G. E. (1991) Factor structure of DSM-III-R person- ality disorders shown by self-report questionnaire: implications for classifying and assessing personality disorders. Acta Psychiatrica Scandinavica, 84, 555-560.
HuNT, C. \& ANDREWs, G. (1992) Measuring personality disorder: the use of self-report questionnaires. Journal of Personality Disorders, 6, 125-133.
Morley, S. \& SNatth, P. (1989) Principles of psychological assessment. In Research Methods in Psychiatry (eds C. Freeman \& P. Tyrer), pp. 133-147. London: Gaskell.
Prohl, B., Coryell, W., Zmmerman, M., el al (1987) Prognostic validity of self-report and interview measures of personality dis- order in depressed inpatients. Journal of Clinical Psychiatry. 48, 468-472.

Department of Psychiatry

University of Cambridge

Addenbrooke's Hospital

Cambridge CB2 $2 Q Q$

\section{Combination treatment of depression}

SIR: The interesting paper by Seth et al (Journal, October 1992, 161, 562-565) can be supplemented by three other investigations which have reported the benefit of combined treatment with selective 5-HT uptake blockers (SSRIs) and tricyclic antidepressants (TCAs) in depressed patients (Weilburg et al, 1989, 1991; Nelson et al, 1991). None of these investigations has been controlled, but effects of combination treatment in patients unresponsive to first-line antidepressant medication are likely to be observations of clinical significance.

For those who may intend to use this drug combination it is important to take note that SSRIs can produce striking increases in plasma concentrations of co-administered TCAs. There have, for example, been reports of severe adverse reactions, including seizures, when fluoxetine has been combined with TCAs (Aranow et al, 1989; Preskorn et al, 1990). It may be that other SSRIs, such as sertraline, would be less likely to increase plasma TCA levels, but until formal studies are available, caution is needed when SSRIs and TCAs are combined. In this respect, the low doses of nortriptyline used by Seth et al are 
undoubtedly wise, but measurement of plasma TCA levels is also a sensible precaution, particularly in the elderly.

Our own experience of combining SSRIs with TCAs such as desipramine and lofepramine has also been encouraging, although sadly the $100 \%$ success noted by the authors has eluded us. An adverse effect we have sometimes encountered when combining SSRIs and TCAs has been an increase in agitation and restlessness. Such reactions are important to recognise in depressed patients and we were therefore interested to note that a similar reaction may have occurred in one of the authors' reported cases. At present we believe that combined SSRI-TCA treatment should be reserved for patients who do not respond to lithium augmentation of first-line antidepressant treatment. However, a significant number of patients who do not respond to the latter approach may be helped by subsequent combination of SSRIs and TCAs.

\footnotetext{
Aranow, R. B., Hudson, J. I., PoPE, H. G., Jr., el al (1989) Elevated antidepressant plasma levels after addition of fluoxetine. American Journal of Psychiatry, 146, $911-913$.

Nelson, J. C., Mazure, C. M., Bowers, M. B., el al (1991) A preliminary, open study of the combination of fluoxetine and desipramine for rapid treatment of major depression. Archives of General Psychiatry, 48, 303-307.

Preskorn, S. H., Beber, J. H., Paul, J. C., et al (1990) Serious adverse effects of combining fluoxetine and tricyclic antidepressants. American Journal of Psychiatry, 147, 532.

Weilburg, J. B., Rosenbaum, J. F., Btederman, J., et al (1989) Fluoxetine added to non-MAOI antidepressants converts nonresponders to responders: a preliminary report. Journal of Clinical Psychiatry, 50, 447-449.

-, -, Meltzer-Brody, S., et al (1991) Tricyclic augmentation of fluoxetine. Annals of Clinical Psychiatry, 3 209-213.
}

P. J. COWEN

Psychopharmacology Research Unit

A. C. POWER

Littlemore Hospital

Oxford $O X 44 X N$

SIR: I read with interest the article by Seth et al (Journal, October 1992, 161, 562-565). They concluded that the combination therapy with nortriptyline and a 5-HT reuptake inhibitor was more effective than individual therapies alone. From their case descriptions, however, I could not find the evidence that the effect of nortriptyline alone was assessed sufficiently before the combination. Moreover, plasma nortriptyline levels in their patients $(118 \mu \mathrm{g} / \mathrm{l}$ in Case 4, $68 \mu \mathrm{g} / \mathrm{l}$ in Case 7, and $104 \mu \mathrm{g} / \mathrm{l}$ in Case 8) were within the reported therapeutic range $(50-139 \mu \mathrm{g} / 1$ by Åsberg et al, 1971; $50-175 \mu \mathrm{g} / 1$ by Kragh-Sørensen, 1973). Thus, there is a possibility that the improvement of their patients was due to nortriptyline alone despite the combination with other drugs.

ÅsBerg, M., Crönholm, B., SJöQvist, F., et al (1971) Relationship between plasma level and therapeutic effect of nortriptyline. British Medical Journal, iii, 331-334.

Kragh-Sorensen, P., Åsberg, M. \& Eggert-Hansen, C. (1973) Plasma nortriptyline levels in endogenous depression. Lancet, $i$, 113-115.

TAKESHI TERAO

University of Occupational and Environmental Health Iseigaoka 1-1, Yahatanishi-ku

Kitakyushu 807

Japan

Authors' RePly: Professor Terao raises the issue that nortriptyline alone could have produced the beneficial effect and this was not assessed sufficiently. This was an open clinical case study where patients were treated empirically without study design. As most patients had been on tricyclic antidepressants, which act on many neurotransmitter systems, including down-regulation of beta receptors (Cowen, 1990), and as clinically greater efficacy for nortriptyline compared with other tricyclics has not been shown (Mandells, 1968), it is unlikely that nortriptyline alone could have produced the benefit. We still, however, acknowledge the issue raised by Professor Terao and concluded in our paper that a doubleblind clinical trial was necessary.

Drs Cowen and Power make a number of interesting comments associated with potential side effects when using combination treatment with tricyclics and selective serotonin reuptake inhibitors (SSRIs). Firstly, they acknowledge the point already made that there is a need for a controlled trial to evaluate the effect of combination treatment in patients who are unresponsive to first-line antidepressant medication.

The second part concerns pharmacokinetic interaction between SSRIs and tricyclics and we would endorse the view that serum levels of tricyclic antidepressants should be monitored. We would also recommend that, particularly in the elderly, blood pressure should also be monitored, as in our experience a few patients have developed postural hypotension requiring lowering of the tricyclic dose.

Thirdly, they raised the issue of the combination treatment producing an increase in agitation and restlessness in some patients, and indeed this has also been our experience. It is, however, notable that SSRIs alone can produce a similar side-effect and, indeed, occasionally a 'serotonergic crisis'. As 